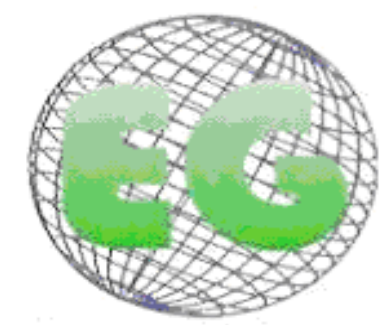

ISSN 1695-6141

$\mathrm{N}^{\circ} 24$

\title{
Rol educativo del profesional de enfermería hacia los familiares de pacientes esquizofrénicos
}

Educational Role of Nursing towards relatives of schizophrenic patients

\author{
*Sifuentes Contreras, A., **Sosa Gil, E., ***Jaimez, T., ${ }^{* * *}$ Manzanillo, Y. \\ * Profesora Asociado de la Clínica de Enfermería Médico Quirúrgica. Departamento Médico Quirúrgico, \\ Escuela de Enfermería, Universidad de Los Andes. **Profesora Titular de la Clínica de Enfermería en Salud \\ Mental y Psiquiatría. Departamento Médico Quirúrgico, Escuela de Enfermería, Universidad de Los Andes. \\ ***Enfermera Asistencial. Hospital Psiquiátrico de Maracaibo. Venezuela.
}

Palabras clave: Rol educativo; Enfermería; Familiares y pacientes esquizofrénicos.

Keywords: Educational Role; Nursing; relatives schizophrenic patients.

\section{RESUMEN}

El objetivo fue determinar el rol educativo del profesional de enfermería hacia los familiares de pacientes esquizofrénicos recluidos en el Hospital Psiquiátrico. Maracaibo, Venezuela. Septiembre 2009- Febrero 2010. El estudio fue descriptivo, con diseño transversal, con una población de 30 familiares, a quienes se les aplicó un cuestionario de 20 ítems. El análisis e interpretación de los resultados se realizó con base a la Estadística Descriptiva.

En relación a la dimensión Promoción de la Salud, se determinó, según la opinión de los familiares, que más de la mitad del personal de enfermería le ha informado sobre la enfermedad mental que padece su familiar; un alto porcentaje de la población estudiada manifestó que las carteleras que se encuentran distribuidas en el ambulatorio no ofrecen información sobre los últimos adelantos científicos en el campo de la psiquiatría; asimismo, más de la mitad de la población afirmó que durante el tiempo que permaneció de visita, el personal de enfermería no le ofreció información científica relacionada con la importancia de reconocer los síntomas de la enfermedad mental de su familiar y expresaron además que este personal sí les informó sobre el tratamiento farmacológico y los efectos que ellos producen; pero no les explicó sobre la dieta alimentaria, más de la mitad de los familiares manifestaron que el personal de enfermería no les informó sobre sus deberes y derechos.

En cuanto a la dimensión Psicosocial, los familiares encuestados afirmaron que el personal de enfermería no expresó preocupación por el estado de salud de su familiar, ni les ayudó a expresar sus temores; tampoco proporcionó manifestaciones de apoyo a través del contacto físico, no demostró preocupación ni interés a sus problemas familiares. Sin embargo, se destacó la alta frecuencia de respuestas positivas en cuanto a que el personal de enfermería sí les permitió la libre expresión de sus sentimientos, se presentó por su nombre, les informó sobre los procedimientos médicos que se les realizan a su familiar, si respetan su condición social, sí ofreció información sobre los cuidados en pro de la salud de su familiar, se sienten satisfechos por la atención que recibe su familiar. 
En relación a la dimensión Necesidades Espirituales, los familiares afirmaron que el personal de enfermería sí respeta sus creencias religiosas.

$\mathrm{Y}$, por último, en un alto porcentaje, expresaron que este personal respeta sus creencias y costumbres culturales.

\section{ABSTRACT}

The core aim of the study was to determine the role of nurses' education towards the families of schizophrenic patients at the Psychiatric Hospital. Maracaibo, Venezuela. Period: September 2009 - February 2010. The design was cross-sectional, with a target population of 30 families, who answered a questionnaire of 20 items. The analysis and interpretation of the data was based on descriptive statistics. Results: In relation to the health promotion dimension, more than half the nursing staff informed them about mental illness suffered by their relatives. A high percentage of the population studied stated that informative billboards, distributed at the hospital, did not provide information on the latest scientific advances in the field of psychiatry. In addition, more than half of the population reported that during the visits, nurses did not give them scientific information about the importance of recognizing symptoms of mental illness. Also, they expressed that the nursing staff explained to them about drug treatment and their effects, but they did not give them diet recommendations. More than half of the relatives stated that nursing staff did not inform them about patients' rights and duties. Regarding the psychosocial dimension, respondents said that nursing staff did not notify them about the health of their patients and they did not help them to express their fears or manifestations of support through physical contact nor did they show concern or interest in their patients' problems. However, there was a high frequency of responses stating that nursing staff did allow free expression of their feelings and provided information about medical procedures and specific measures of healthcare. They were satisfied with the healthcare provided by nurses. In relation to spiritual needs, the relatives said that the nursing staff showed respect for their religious beliefs. Finally, a high percentage of people expressed that nurses respected their beliefs and customs.

\section{INTRODUCCIÓN}

En la salud de las personas el grupo social que más influye es la familia, es una de las instituciones sociales más antigua y más fuerte. Sus miembros tienen una historia común, comparten rasgos genéticos, entorno, costumbres, creencias, actitudes generales y estilos de vida. Al considerar la familia como un sistema, se constata que funciona como una unidad, ya que a través de las interacciones que se generan entre sus miembros y la dependencia mutua que existe entre ellos, los factores que influyen en uno de sus miembros afectan a todos los demás en mayor o menor grado.

La familia, a través de la historia, ha sido siempre la principal fuente de apoyo y cuidados, estos son proporcionados por los diferentes miembros de la unidad familiar. El estado de salud de un miembro de la familia y su reacción frente a la enfermedad influyen en los mecanismos físicos y psicológicos de soporte que actúan dentro de la familia, y reciben, a su vez, influencia de estos mecanismos. Las modificaciones que conlleva la vida moderna han producido cambios que nunca se habían previsto en las necesidades de salud y en la demanda de una mayor cobertura en cuanto a los servicios de la misma. La enfermedad, la discapacidad y situaciones como el desempleo puede alterar el equilibrio de un grupo y afectar la salud mental y física de sus miembros.

En 1994 la reunión del Comité de Expertos en Enfermería de la Organización Mundial de la Salud (OMS), formuló la siguiente declaración: Los servicios de enfermería orientados a la salud de la familia se basan en la concepción de ésta como unidad y tienen por objeto atender las necesidades y preocupaciones de la familia en materia de salud, animándola a utilizar sus propios recursos, humanos y materiales, y señalando la manera óptima de utilizar los servicios de salud disponibles. ${ }^{1}$ Sin embargo, esta concepción de la familia debe ampliarse, ya que los cambios que se van sucediendo en el mundo actual nos obligan a ser "abiertos" y flexibles, considerando miembros de la misma, no sólo a las personas que 
comparten un mismo apellido o lazos de sangre, o que viven en el mismo hogar, sino también a los grupos que comparten el sentimiento de "sentirse familia". Ésta concepción del núcleo familiar permite al personal de enfermería, en su rol educativo ayudar a todos sus miembros en la consecución de mejores niveles de salud; igualmente no se puede olvidar que la familia es la unidad básica de atención de la enfermería comunitaria; a través de la misma hace extensibles sus cuidados a la comunidad y al individuo.

La Organización Mundial de la Salud, en su informe sobre la salud en el mundo, se refiere a la salud mental en términos de "nueva comprensión, nueva esperanza". Así, dicho informe vierte nueva luz en trastornos mentales y ofrece nueva esperanza a las personas que sufren enfermedad mental y a sus familias. ${ }^{2}$ Este mismo documento advierte que los problemas mentales, neurológicos o del comportamiento, afectan a 450 millones de personas en todo el mundo, se estima que alrededor de un $25 \%$ de la población mundial podría padecer algún trastorno mental a lo largo de su vida. Así mismo, prevé que los trastornos mentales lleguen a ser la principal causa de años de vida perdidos ajustados por discapacidad, fundamentalmente a expensas de los trastornos depresivos.

Por otra parte, el informe sobre la salud en el mundo, presentado por la OMS, el Banco Mundial y la Universidad de Harvard reportó que la esquizofrenia se encuentra entre las diez enfermedades que representan una "Carga Mundial de Enfermedad". Al valorar los "Años de Vida Perdidos Ajustados en función a Discapacidad", la esquizofrenia se colocó en el cuarto lugar dentro de los grupos diagnósticos más frecuentes. Así mismo, la esquizofrenia se encuentra entre las diez primeras causas de incapacidad en la población de edades comprendidas entre 18 y 44 años. ${ }^{3}$

En el mismo documento destacan que las características de la enfermedad, edad de inicio, evolución y pronóstico, reducen las posibilidades de acceso al empleo, el cuidado de uno mismo y afecta la capacidad de relación, siendo el paciente susceptible de requerir cuidados, tanto de tipo formal como informal para lograr un adecuado mantenimiento de su salud. En muchos casos, provoca dificultades en el desempeño de las funciones propias de cada etapa del ciclo vital, imposibilitando el logro de una vida independiente. En este orden de ideas, es importante mencionar que las causas de las enfermedades mentales se encuentran principalmente en las relaciones familiares enfermizas, ya que la vida familiar es una fuerza ambiental de tal importancia que puede afectar en forma determinante el curso, la aparición y el pronóstico de las enfermedades mentales. ${ }^{4}$

Al respecto la OMS sostiene que la enfermedad mental de un miembro de la familia se encuentra ligada inexplicablemente a la salud de los otros miembros, quienes influencian y son influenciados por aquel que padece la enfermedad. Sin embargo, existen aspectos que alteran la salud integral de los miembros de la familia en su estructura y funcionamiento lo que acarrea la disfuncionalidad de los roles y operatividad del núcleo familiar. ${ }^{5}$ Asimismo, El Consejo Internacional Enfermería (CIE) y las Asociaciones Nacionales de Enfermeras, se sienten profundamente preocupados por la calidad y la continuidad de los tratamientos y los cuidados dispensados a las personas que padecen desórdenes mentales y de la conducta. Esta preocupación está motivada también por las necesidades de sus familias y comunidades, y por el estigma y la discriminación que sufren las personas afectadas por desórdenes de la salud mental, y quienes las cuidan, entre ellos, los profesionales de salud.

Los señalamientos anteriores indican que, normalmente, la familia debe ocuparse de la persona enferma más allá de lo que suponen sus funciones habituales, convirtiéndose en agentes terapéuticos con los que hay que trabajar en el establecimiento de objetivos, prioridades y tareas, reforzando sus potencialidades, para abordar problemas como parte de 
una estrategia global de tratamiento. En este contexto, los pacientes esquizofrénicos se han considerado el grupo diana de la reforma psiquiátrica y el grupo más numeroso para los que se ha transferido la atención desde el hospital a los servicios de salud mental comunitarios.

Tomando en consideración el documento de la OMS, donde propone un modelo basado en la participación, en el cual los enfermos y la familia tengan la posibilidad de reestructurar su condición ante la sociedad, propone también que los profesionales de la salud, en especial enfermería, deben asumir la labor de orientarles, motivarles y situarles ante el sistema de vida propiciando la integración social $y$, principalmente la relación educativa con los familiares. $^{3}$

Actualmente, la esquizofrenia constituye uno de los trastornos mentales más complejos y una de las enfermedades de considerable impacto en la salud pública mundial. Morrinson, la define como: "La presencia de alucinaciones, ideas delirantes, desorganización del pensamiento y de la conducta, así como empobrecimiento afectivo y deterioro cognitivo". Acompañada de deterioro grave en el área de la personalidad y de la actividad social que repercute tanto en el entorno familiar, como en la sociedad, afectando las relaciones entre el paciente y su ambiente. ${ }^{6}$

Tras lo expuesto en el apartado anterior, en el Hospital Psiquiátrico de Maracaibo los programas educativos emanados por el Ministerio del Poder Popular para la Salud, se han cumplido esporádicamente y en la actualidad se cumple un programa denominado "Atención para Esquizofrénicos y sus Familiares"; el mismo tiene como objetivo integrar al usuario, familia y comunidad en el conocimiento de las enfermedades mentales para fortalecer el bienestar del enfermo mental. Las reuniones de terapia se efectúan el último viernes de cada mes, bajo la asesoría de un médico psiquiatra y una trabajadora social. Es importante señalar que, muchas veces, este programa educativo se cumple con algunas limitaciones, posiblemente por factores como: inasistencia de los familiares de los pacientes a la programación establecida para estos encuentros, la distancia geográfica que separa a muchos de ellos del sitio de reunión y las actividades laborales y familiares, entre otros.

Lo planteado en el parágrafo anterior, refleja la ausencia del rol educativo de los Profesionales de la Enfermería en la participación en los programas didácticos dirigidos a los familiares. Ahora bien, a lo largo de la trayectoria laboral y profesional de las autoras en el Hospital Psiquiátrico de Maracaibo, se ha observado con frecuencia que el personal de enfermería que por razones institucionales no están integrados (as), en el equipo de salud de las reuniones de terapias, los días de visitas desempeñan un rol educativo respondiendo a preguntas e inquietudes que hacen los familiares acerca de la enfermedad mental, tratamiento y cómo abordarlos en el hogar en el momento que ellos presentan su sintomatología. Por otro lado, se ha observado que los enfermos mentales al reintegrarse a su vida familiar, al poco tiempo reingresan, de nuevo, a la institución hospitalaria. En vista de la situación que cotidianamente se evidencia en los centros de salud de la región y el país, se decidió emprender esta investigación con el objetivo de determinar el rol educativo del profesional de enfermería hacia los familiares de pacientes esquizofrénicos del Hospital Psiquiátrico de Maracaibo, Estado Zulia. Periodo Septiembre 2009- Febrero 2010.

En tal sentido, se trazaron los siguientes objetivos específicos dentro de la investigación:

- Identificar las actividades educativas relacionadas con la promoción de la salud que realiza el Profesional de Enfermería en los familiares de los pacientes esquizofrénicos en el Hospital Psiquiátrico. 
- Identificar las actividades educativas relacionadas con las necesidades psicosociales que realiza el Profesional de Enfermería en los familiares de los pacientes esquizofrénicos en el Hospital Psiquiátrico

- Identificar las actividades educativas relacionadas con las necesidades espirituales que realiza el Profesional de Enfermería en los familiares de los pacientes esquizofrénicos en el Hospital Psiquiátrico.

\section{METODOLOGÍA}

El trabajo se inscribió dentro del tipo de estudio descriptivo, además, se correspondió con un diseño de campo, de corte transversal, el período de investigación estuvo comprendido entre Septiembre 2009- Febrero 2010, en una realidad especifica, en este caso los familiares que asisten al Hospital Psiquiátrico Maracaibo, todo ello tomando en consideración que "los diseños de corte transversal son recolectados en un solo momento, en un tiempo único, cuyo propósito es describir variables y analizar su incidencia e interacción en un momento dado". ${ }^{7}$ La población o universo está referida a "la totalidad del fenómeno a estudiar, donde las unidades de población poseen una característica común, la que se estudia y da origen a los datos de la investigación". ${ }^{8}$

De acuerdo a la definición anterior, en esta investigación la población de estudio estuvo representada por treinta (30), familiares quienes acudieron a las visitas de su familiar hospitalizado durante el periodo de recolección de datos en el Hospital Psiquiátrico de Maracaibo. En este sentido y atendiendo al reducido grupo de familiares que conforman la población en estudio, la cual por sus características de ser finita, hizo que no fuese necesario la selección de una muestra, por lo tanto se trabajó con la totalidad. Al respecto, "es recomendable encuestar a la población de manera total cuando comprende menos de cien unidades de análisis"

El instrumento utilizado para la recolección de datos fue un cuestionario conformado por veinte (20), ítems, estructurado en tres (3), dimensiones: Promoción de la Salud, Necesidades Psicosociales y Necesidades Espirituales. Se define el cuestionario "como el instrumento que agrupa una serie de preguntas relativas a un evento o temática particular, sobre el cual el investigador desea obtener información, las preguntas se hacen por escrito, deben cumplir los requisitos de validez y de confiabilidad" ${ }^{10}$. El instrumento diseñado fue sometido a la prueba de validación por medio de la validez del contenido, o juicio de expertos; estos evaluaron la construcción del instrumento, desde diversos aspectos como coherencia, viabilidad, claridad y congruencia; estos validadores concluyeron que el mismo reunía las condiciones de validación. Una vez aplicado el instrumento y tabulados manualmente los datos, fueron analizados utilizando la estadística descriptiva.

\section{RESULTADOS}

En la dimensión promoción de la salud, se determinó que el 55,6\% de la población estudiada manifestó que la (el) enfermera(o), le ha informado sobre la enfermedad mental que padece su familiar. Un $81.5 \%$ afirmó que las carteleras que se encuentran distribuidas en el hospital no cuentan con información acerca de los últimos adelantos científicos en el campo de la psiquiatría. El $77.8 \%$ opinó que durante el tiempo que permanecieron visitando a su familiar el personal de enfermería no le ofreció información científica de la importancia de reconocer los síntomas de la enfermedad mental de su familiar. $70.4 \%$ de los familiares expresó que 
durante el tiempo que permanecieron de visita las enfermeras y los enfermeros les explicaron sobre la importancia del tratamiento farmacológico y los efectos que ellos producen. Pero el 63\% afirmó que durante la estadía de visita el personal de enfermería no les explicó sobre la dieta alimenticia que debe recibir su familiar al ser dado de alta. Por otra parte, el 59\% manifestaron que el personal de enfermería impartió los cuidados de higiene que debe recibir su familiar. El 62.97\% declaró que desconocen los derechos del enfermo mental a ser tratado en todo momento con la solicitud, el respeto y la dignidad propios de su condición de persona. Respecto a la dimensión necesidades psicosociales, el 55.6\% afirmó que el personal de enfermería no les expresó preocupación por el estado de salud de su familiar. Un $66.7 \%$ opinó que el personal de enfermería es insuficiente para cubrir las necesidades de atención de enfermería que requieren los pacientes recluidos. El $56 \%$ de los familiares opinó que el personal de enfermería no ofreció manifestaciones de afecto a través del contacto físico. Se verificó que el $56 \%$ de dicho personal no les ofreció apoyo cuando se sentían preocupados por el pronóstico de su familiar.

Por otra parte, se encontró en un $70.4 \%$ de las respuestas emitidas por los familiares encuestados estos afirman que este personal les permitió la libre expresión de sus sentimientos. El 66,66\% opinó que el personal de enfermería no le dedicó tiempo para conversar sobre la evolución que ha tenido su familiar hospitalizado en el psiquiátrico. Los familiares opinaron en un $70.4 \%$, se presentó y dio a conocer su nombre para identificarse con ellos. El $51.8 \%$ respondió que le informó oportuna y verazmente los procedimientos médicos que se le realizaron a su familiar durante la hospitalización. Se verificó que el $88.9 \%$ afirmó que la(el) enfermera(o) respeta su condición social. El 62.97\% expresó que les ofreció información acerca de los cuidados que debe realizar en el hogar en pro de la salud de su familiar. Con relación a si se sienten satisfechos por la atención que recibe su familiar por parte del personal de enfermería, el $70.4 \%$ afirmó su complacencia. En cuanto a las necesidades espirituales, se determinó en un $88.9 \%$ afirmó que el personal de enfermería que labora en el hospital psiquiátrico respeta sus creencias religiosas, el $85 \%$ de la población estudiada, sus costumbres culturales.

\section{DISCUSIÓN}

Con base a los resultados expuestos en cuanto a la Promoción de la Salud, es considerada como uno de los pilares de la acción de la salud pública; es una estrategia transformadora que involucra al individuo y a su medio social y tiene como objetivo proporcionar los medios necesarios para mejorar su salud y ejercer un mayor control sobre la misma. Gallar, define promoción de la Salud como "El conjunto de medios y estrategias encaminados a procurar la adopción de unos hábitos de vida saludable". ${ }^{11}$ Este concepto está íntimamente vinculado al de Educación para la Salud, que el citado autor lo concibe como "Un proceso planificado y sistemático de comunicación y de enseñanza-aprendizaje orientado a hacer fácil la adquisición, elección y mantenimiento de las prácticas saludables, y a hacer difíciles las prácticas de riesgo". Así pues, la educación para la salud es una estrategia inherente a la promoción de la salud. Es necesario que la familia tome conciencia de que la esquizofrenia puede ser controlada. Para ello, es necesario establecer estrategias de educación a los familiares directos de estos pacientes en cuanto a: causas de la patología, identificación de signos y síntomas, alimentación, higiene y sobre todo el tratamiento que suele combinar la farmacoterapia, la psicoterapia grupal y familiar y la participación de grupos de apoyo. En este orden de ideas, es necesario que los familiares cumplan con el tratamiento ya que dejar de tomar la medicación implicaría una nueva recaída, un nuevo brote y el consecuente empeoramiento de la enfermedad; de ahí la importancia, una vez más, de la ayuda y cooperación de la familia. En este sentido, el profesional de enfermería puede y debe 
conocer las habilidades elementales de la educación para la salud, ya que ésta es un instrumento esencial para la consolidación de los hábitos de vida saludable.

Con respecto a los resultados sobre las Necesidades Psicosociales, González señala que los objetivos primordiales de los cuidados de Enfermería al familiar están dirigidos a:

a) Facilitar la comunicación familia - enfermera(o);

b) integrar al familiar a la nueva situación de forma gradual;

c) aumentar los recursos familiares, conocimiento sobre la enfermedad, tratamiento y efectos secundarios;

d) capacitar para afrontar las crisis de forma adaptativa;

e) fomentar la participación en los cuidados;

f) mejorar el deterioro social y reducir la carga y la emoción expresada en la familia. ${ }^{12}$

De acuerdo al parágrafo anterior es preciso señalar que "La comunicación es un medio que permite al individuo comprender a otro, aceptar y ser aceptado, transmitir y recibir información, dar y recibir instrucciones, enseñar y aprender". Por lo tanto, el profesional de enfermería se comunica con el usuario y viceversa. La comunicación siempre es un proceso de dos vías, interactivo y relevante, donde toda forma expresiva del profesional es fundamental, y dentro de su contexto existe la intención de ayuda y proporcionar cuidados, lo que se deriva de una comunicación de carácter terapéutico, haciendo alusión al apoyo psicológico y a la ayuda en la satisfacción de las necesidades humanas. ${ }^{13}$

El entrenamiento en habilidades sociales, conjuntamente con el tratamiento farmacológico, suele ser útil y de apoyo para el paciente; el esquizofrénico ve muy afectada su relación con los demás, como consecuencia de su escaso contacto ocular, demora en las respuestas, extrañas expresiones faciales, pérdida de la espontaneidad y de la afectividad y percepciones poco precisas. Por ello el uso de videos del paciente o juego de roles durante la terapia ayudan a éste a una mejor socialización. ${ }^{14}$

Las terapias familiares constituyen un medio de gran utilidad en la orientación, integración y rehabilitación del paciente. La familia del paciente esquizofrénico es una fuente inestimable de información tanto para establecer el diagnóstico como para contribuir en el comportamiento y socialización del paciente. Educar a la familia acerca de la enfermedad ayuda a plantearles expectativas razonables, mejorando notablemente el curso de la misma $^{14}$

En relación a las necesidades Espirituales, es de vital importancia que el profesional de enfermería mantenga respeto por el usuario, ya que es uno de los elementos de la relación terapéutica, imprescindible para garantizar una relación de ayuda, mejorando la autoestima sin juzgarle, sin poner condiciones a su dignidad personal. Por lo tanto el "Respeto es un valor propio de cada persona que ha de estar implícito en cualquier relación y que permite una aceptación global e incondicional"12 El Profesional de Enfermería, debe respetar al usuario como una persona de valía y dignidad. Es importante señalar que todo ser humano merece ser tratado con nobleza, respetando sus derechos e ideologías. Para que la relación entre el profesional de enfermería, el usuario y sus familiares sea eficaz es vital hacer valer plenamente sus derechos. Para Peplau, "La enfermería es un instrumento educativo, una fuerza de maduración que ayuda a promover en la personalidad el movimiento de avance hacia una vida creativa, constructiva, productiva, personal y comunitaria" y por ello describió 4 fases para conceptuar el proceso de interrelación personal: orientación, identificación, aprovechamiento y resolución, aspectos tomados en cuenta en la variable de estudio la cual se dimensiona en dos aspectos: la comunicación y el apoyo emocional. Los cuidados en 
Enfermería Psicodinámica exigen ser capaz de comprender la propia conducta para poder ayudar a otros a identificar las dificultades percibidas y aplicar principios de relaciones humanas a los problemas que surgen en todos los niveles de experiencia, basándose en la comunicación, como un importante proceso interpersonal y terapéutico que funciona en términos de cooperación con otros procesos humanos que hacen de la salud una posibilidad para las personas. ${ }^{15}$

El componente espiritual es el sentimiento de conexión entre el enfermero y el paciente, es la sensación de sentirse parte de algo más que uno mismo. Cuando se desatiende el componente espiritual tanto enfermeros como usuarios pueden sentirse desconectados y aislados. El profesional de enfermería debe servir de modelo para adquirir patrones diferentes de funcionamiento, que aumenten la autonomía y capacidad adaptativa.

\section{CONCLUSIONES}

Después de haber analizado los resultados obtenidos con la aplicación del instrumento dirigido a determinar, el rol educativo del profesional de enfermería hacia los familiares de pacientes esquizofrénicos del Hospital Psiquiátrico de Maracaibo, Estado Zulia. Periodo Septiembre 2009- Febrero 2010. Se bosquejan las siguientes conclusiones:

En relación a la dimensión promoción de la salud, se determinó según la opinión de los familiares, que más de la mitad del Personal de Enfermería le ha informado sobre la enfermedad mental que padece su familiar. Un alto porcentaje de la población estudiada manifestó que las carteleras que se encuentran distribuidas en el ambulatorio no ofrecen información sobre los últimos adelantos científicos en el campo de la psiquiatría. Asimismo, más de la mitad de la población afirmó que durante el tiempo que permaneció de visita la enfermera(o), no les ofreció información científica de la importancia de reconocer los síntomas de la enfermedad mental de su familiar y expresaron además que sí les informó sobre el tratamiento farmacológico y los efectos que ellos producen; pero no les explicó sobre la dieta alimentaria. Y por último, más de la mitad de los familiares manifestaron que el personal de enfermería no les informó sobre sus deberes y derechos.

En cuanto a la dimensión Psicosocial, los familiares encuestados afirmaron que el personal de enfermería no les expresó preocupación por el estado de salud de su familiar; perciben que este personal no es suficiente para cubrir las necesidades de salud de su familiar, no les ayudó a expresar sus temores, tampoco ofreció manifestaciones de apoyo a través del contacto físico, ni demostró preocupación o interés a sus problemas familiares. Sin embargo, se destacó la alta frecuencia de respuestas positivas en cuanto a que el personal de enfermería sí les permitió la libre expresión de sus sentimientos, se presentó por su nombre, les informó sobre los procedimientos médicos que se le realizan a su familiar, respetan su condición social, les ofrecen información sobre los cuidados en pro de la salud de su familiar y se sienten satisfechos por la atención que recibe su familiar.

En relación a la dimensión Necesidades Espirituales, los familiares encuestados afirmaron que el Personal de Enfermería sí respeta sus creencias religiosas; un alto porcentaje de los encuestados expresaron que el Personal de Enfermería respeta sus creencias y costumbres culturales.

\section{Recomendación}

El Personal de Enfermería que labora en el hospital psiquiátrico, debe fortalecer los aspectos positivos de la relación terapéutica con los familiares de los pacientes recluidos en 
esta unidad, a través de talleres, charlas alusivas en relación a la promoción de la salud y actualización en el cuidado enfermero.

\section{BIBLIOGRAFÍA}

1. De la Revilla, L. (1996). Conceptos e Instrumentos de la Atención Familiar. Barcelona: Doyma

2. Organización Mundial de la Salud. (2001). La Salud Mental en el Mundo. [Documento en línea] Disponible: http://www.ome-aen.org/OMS.htm. [Consulta: 2009, Octubre 5].

3. Alfonso, A. (2006). Valoración de las Necesidades del Paciente Esquizófrenico ante la Pérdida del Cuidador Principal. España. [Documento en línea] Disponible: http://ciencia.micinn.fecyt.es/universidades/dcs/files/Salud-Mental.pdf [Consulta: 2009, Octubre 2].

4. Cordero, J. (1999). La Familia: Problemas de Salud. México: Mc Graw Hill. Interamericana.

5. Organización Mundial de la Salud. (1999). Los Desordenes Mentales en la Atención Primaria. [Documento en línea] Disponible: http://www.omeaen.org/OMS.htm. [Consulta: 2009, Octubre 5].

6. Morrinson, M. (1999). Fundamentos de Enfermería en Salud Mental. España: Harcourt Brace.

7. Hernández, R., Hernández, C y Baptista, P. (2007). Metodología de la Investigación. (3ª ed). México: McGraw-Hill Interamericana.

8. Tamayo, M. (1998). El Proceso de la Investigación Científica. (3ª̣.ed). México: Limusa.

9. Münch, L. y Ángeles, E. (1997). Métodos y Técnicas de Investigación. México: Trillas.

10. Hurtado de Barrera, J. (2000). Metodología de la Investigación Holística. (2 ${ }^{\underline{a}}$ ed). Caracas: SYPAL.

11. Gallar, M. (2006). Promoción de la Salud y Apoyo Psicológico al Paciente. (4 ${ }^{\mathrm{a}}$ ed). España: Thomson. [Libro en línea] Disponible: http://books.google.com/books?id=tqpZwfbPdklC\&pg=PA317\&lpg=PA317\&dq=promocion+ de+la+salud\&source=web\&ots=0JlbWs99N1\&sig=gf2rYEf8plynrOzvjLX1stEBXjU\#PPP5,M 1. [Consulta: 2009, Enero 5].

12. González, J. (2005). Enfermería Principios y Práctica. Argentina: Interamericana.

13. Dugas, B., (1994). Tratado de Enfermería Practica. (4⿳亠丷a ed). México: Manual Moderno.

14. Sosa, E. (2004). Enfermería en Salud Mental y Psiquiatría. Módulo Autoinstruccional. Departamento de Publicaciones. Programa de Profesionalización. Escuela de Enfermería. Universidad de Los Andes. Mérida. Venezuela.

15. Ruby, L Wesley, N (1995). Teorías y Modelos de Enfermería. (2ª ed). México: Mc Graw Hill. Interamericana.

ISSN 1695-6141

@ COPYRIGHT Servicio de Publicaciones - Universidad de Murcia 